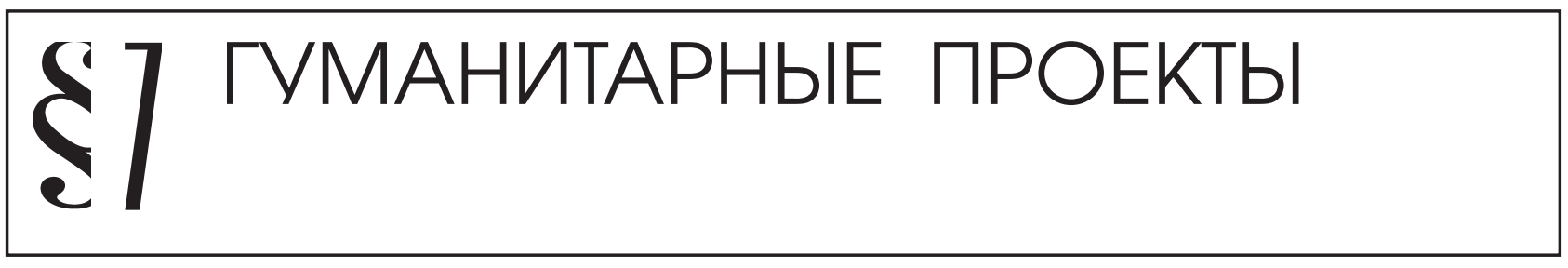

В.В. Насонкин

\title{
ПОЛИТИЧЕСКОЕ ЗНАЧЕНИЕ АВТОНОМИИ ОБРАЗОВАТЕЛЬНОГО УЧРЕЖДЕНИЯ В ФОРМИРОВАНИИ ЭФФЕКТИВНОЙ МНОГОУРОВНЕВОЙ СИСТЕМЫ УПРАВЛЕНИЯ ОБРАЗОВАНИЕМ
}

\begin{abstract}
Аннотация: Статья посвящена проблеме расширения автономии школьных учреждений и ее влияния на результаты обучения. Выявляется взаимосвязь между автономией в сфере распоряжения ресурсами, определения содержания образования и форм контроля над знаниями учащихся, а также опубликованием результатов работы конкретных школьных учреждений и повышением эффективности их работы. В целом констатируется неоднозначность автономии школьного учреждения как фактора повышения конечных результатов его деятельности.
\end{abstract}

Ключевые слова: Политология, автономия, публичность результатов, эффективность, ресурсы, система управления, образовательное учреждение, ОЭСР, доступность, школа

$\mathrm{B}$ настоящее время все страны, в первую очередь, страны-члены ЕС и Организации экономического сотрудничества и развития (далее ОЭСР) подчеркивают значение человеческого капитала для интенсивного социальноэкономического развития. При этом по оценкам специалистов каждый пятый учащийся в странах ОЭСР не получает минимального уровня образования. Кроме того, учащиеся из малообеспеченных и неблагополучных семей в два раза чаще имеют плохую успеваемость. Недостаточный уровень справедливости и включенности в учебный процесс может привести к их неуспеваемости, что приведет к выбыванию из школы каждого пятого молодого человека до момента получения им полного общего образования [1].

Данная проблема будет нами рассматриваться в отдельном материале, но она тесно связана с другой проблемой, которая вызывает многочисленные острые дискуссии: как обеспечить организационно эффективность системы образования, призванной формировать высокое качество человеческого капитала в сложнейших условиях изменившихся внешней среды, психологии челове- ка, требований к его образовательному уровню и мыслительной деятельности.

При этом основной акцент переместился в последние годы с простого обеспечения доступности образования для всех на повышение качества образования и приведение в соответствие его содержания и объема потребностям общества. Именно это изменение базовой цели функционирования системы образования резко повысило уровень неопределенности, т.к. гораздо проще оценивать масштаб системы и его изменения, чем измерять качество функционирования и эффективность управления. Наибольший уровень неопределенности возник в связи с изменениями институциональной структуры, что и привело к неудачам реформ в этой сфере во многих странах.

Автономия на локальном уровне (школы или школьного совета-округа) дискутировалась многократно, тенденции развития в разных странах носили часто противоположный характер: в некоторых странах усиливалась децентрализация, особенно в сфере разработки учебных планов и подборе учителей, в то время как в других - повышалась централизация аналогичных процессов. В наличии 
двух противоположных тенденций содержится коренное противоречие. С одной стороны, сторонники автономии отдельного учреждения считают, что каждая школа в состоянии лучше оценить потребности населения своей территории и, таким образом, лучше понимает стоящие перед ней цели и определяет пути их достижения. Это позволяет лучше распределять ресурсы, повышать результативность работы школ в соответствии с местными условиями. Противоположная тенденция опирается на мнение о недостаточности у отдельной школы способности принятия решений и наличия у всех участников процесса противоречивых намерений по многим направлениям. Соответственно, автономия школ создает риск формирования у каждой отдельной школы целей, отличных от общих направлений развития, и отступления от общих стандартов образования.

Несмотря на противоречивые оценки результатов расширения школьной автономии в ряде развивающихся стран и США, оптимизм в отношении возможностей такого расширения сохраняется ${ }^{1}$. Анализ реально сложившейся ситуации в разных странах, включая европейские, по мнению ряда специалистов, заставляет искать ответы на четыре основных вопроса[2]:

1. Сама концепция широкой автономии на локальном уровне (отдельного школьного учреждения или учредителя) имеет разнообразные формы, что чрезвычайно затрудняет измерение эффективности этого явления. На локальном уровне наиболее эффективно принимаются решения, не требующие стандартизации и четкого определения результатов, в то время как формирование стандартизированных требований должно иметь более общий характер и приниматься на более высоких уровнях управления. Решения о стандартах и базовых требованиях носят исключительно политический характер.

2. Влияние автономии может быть различным на различные элементы системы: знание местных условий может одновременно способствовать

\footnotetext{
${ }^{1}$ Cм.: World Bank. Education for All Initiative // http://en.wikipedia.org/wiki/Education_For_All, 2011; UNESCO. Millennium Development Goals of the UN // http://en.wikipedia.org/wiki/ Millennium_Development_Goals, 2011
}

повышению качества образования или, наоборот, стимулировать оппортунистическое поведение работников школьной системы и вести к утрате системности в учебном процессе. В данном случае воздействие на результаты обучающихся четко коррелирует с уровнем ответственности школы за результаты ее работы, что отражается, в широком смысле слова, на развитии всей системы образования, а значит, на уровне развития страны.

3. При проведении сравнительного анализа не всегда есть возможность вычленить влияние автономии в числе других факторов. Так, если более динамично развивающиеся школы получают автономию или более заинтересованные родители выбирают автономные школы, означает ли это, что автономия оказывает влияние на результаты учащихся.

4. Многие вопросы о разграничении полномочий ставятся на общенациональном уровне, причем для местной автономии остается мало пространства, т.к. разрабатываются национальные стандарты, проводятся унифицированные процедуры оценки работы отдельных школ, вводятся особые процедуры отчетности, а также жестко регулируется, какие именно решения могут приниматься на уровне отдельной школы. При сравнении разных стран эта проблема усложняется особенностями культуры, спецификой системы управления и институционального дизайна.

Анализ деятельности наиболее успешных и быстро развивающихся систем образования, в том числе в рамках широкомасштабного исследования $\mathrm{PISA}^{2}$, позволяет выделить общие черты этих систем, которые заключаются в следующем:

Во-первых, политические и общественные лидеры способны убедить своих соотечественников, что образование является более важным вопросом в развитии страны, чем любой другой. Однако прогресс в сфере образования возможен только в том случае, если учителя, родители и все граждане уверены, что все ученики должны получать
2 Последнее полностью проанализированное исследование
было осуществлено в 2009 году, его результаты обобщены
в публикации, изданной в 2010 году. См.: OECD, PISA 2009
Results: What Makes a School Successful? - Resources, Policies
and Practices // http://dx.doi.org/10.1787/9789264091559-en 


\section{Политика и общество 2 (98) • 2013}

образование мирового уровня. В странах, где сохраняется мнение, что ученики имеют изначально различные потребности в образовании, которые должны удовлетворяться наличием различных типов школ, существует риск формирования серьезного социального расслоения. Наиболее успешными оказываются те, кто стремится обеспечить соответствие системы образования разнообразию способностей, интересов и социальному происхождению учеников с помощью индивидуализации подходов к образовательному процессу.

Во-вторых, успешные системы обладают четкими и достаточно высокими образовательными стандартами, которые действуют во всех элементах системы, ориентированы на более высокие уровни мыслительной деятельности, что позволяет каждому обучающемуся представлять себе высоту «барьера», который необходимо взять дл получения определенной квалификации, причем стандарты включают не только содержание знаний, требуемых для получения искомой квалификации, но и способность применения этих знаний в практической деятельности. Обучающиеся могут перейти на следующую ступень - начать работать или получать образование более высокой ступени - только достигнув необходимого для этого уровня квалификации.

В-третьих, качество образования не может быть выше качества работающих в системе учителей и руководителей школьных учреждений. Многие наиболее успешные страны предоставили работникам системы образования гораздо больше свободы в распределении ресурсов, организации их труда, что не исключает одновременно необходимости эффективного контроля и многоаспектной ответственности школ за качество предоставляемых ими услуг. Это стимулирует учителей к совместной творческой деятельности, оценке работы своих коллег и распространения лучшей практики, используемой наиболее успешными преподавателями ${ }^{3}$.

Как можно видеть из вышесказанного, возможность самостоятельности в деятельности школьных учреждений рассматривается как один важнейших политических факторов в повышении эффективности системы образования в современных условиях. Автономия школы становится од-

${ }^{3}$ CM. OECD, PISA 2009 Results. - P. 4. ним из решающих организационных факторов, влияющих на получаемые в отдельной школе и во всей системе образования результаты.

Как показывает соответствующий раздел исследования PISA, даже с учетом показателя валового национального продукта на душу населения, автономия школы скорее позитивно влияет на получаемые результаты. При этом в большинстве исследований различают два основных направления, по которым школам может предоставляться автономия: во-первых, в сфере определения учебных планов и программ, а также организации и форм оценки результатов обучения и во-вторых, в вопросах распределения ресурсов, в том числе, кадровых, что включает вопросы подбора учителей, организации их труда, оплаты, оценки достижений наиболее успешных учителей.

Корреляции автономии школ в национальных системах образования и результатов обучения (например, компетентность в сфере чтения и понимания текста) неоднозначна. В рамках исследования PISA-2009 были рассчитаны индексы автономии отдельного школьного учреждения в области распоряжения ресурсами по шести важнейшим позициям: право самостоятельно принимать на работу учителей, увольнять учителей, устанавливать исходную заработную плату, определять правила повышения заработной платы, принимать решения по формированию школьного бюджета и распределять средства школьного бюджета на различные цели. Показатели определялись в трех основных вариантах: 1 - основные полномочия в распределении ресурсов принадлежат директору школы и учителям; 2 - полномочия распределены между директором и учителями и органами управления образованием общенационального (федерального) и регионального уровня; 3 - все полномочия принадлежат органам управления образования различных уровней. Среди стран ЕС, по результатам исследования, наибольшей автономией обладают школы Чешской и Словацкой республик, Нидерландов, Дании, Швеции и Великобритании. Их индексы превышают средний уровень стран, участвовавших в исследовании. Как можно видеть, среди вышеназванных стран представлены, с одной стороны, новые демократии, чьи системы образования пережили в последние десятилетия значительную трансформацию, а также северные страны, стремящиеся в цело в наиболее эффектив- 
ной организации деятельности социальной сферы в рамках социал-демократической модели социального государства. Нельзя также не отметить присутствие в составе группы Нидерландов и Великобритании, прошедших радикальные реформы по децентрализации управления в 80-е годы.

В федеративных государствах ЕС - ФРГ, Австрии, Бельгии - индекс существенно ниже ${ }^{4}$.

В Чешской республике и Великобритании максимальной является также автономия школ в определении программы и форм оценки уровня подготовленности учащихся. Важным является при этом уровень ответственности и формы отчетности школ. Наибольшее количество учащихся в школах, которые открыто публикуют и рассылают отчеты о результатах своих учащихся установлено в ЕС в Дании (45\%); в Нидерландах (63\%), в Норвегии (58\%), в Республике Польша, в Словацкой республике (63\%), в Швеции (61\%); в Великобритании (80\%)[3].

В ряде исследований эти противоречия связываются с уровнем экономического развития стран и основными параметрами их систем образования. В более высокоразвитых странах с качественными системами образования автономия отдельных школьных учреждений дает значительные преимущества, в то время как в странах испытывающих экономические трудности и имеющих недостаточно развитые образовательные системы или системы образования, переживающие период масштабной трансформации, автономия может скорее наносить ущерб и создавать дополнительные риски [4].

В последние годы многие страны провели интенсивную децентрализацию политики в области образования, что должно, как считается, повысить качество образования, а также способствовать эффективности процесса управления, стимулировать инновации, противодействовать неравенству и сегрегации в сфере образования[5]. При этом в современных зарубежных исследованиях по вопросам политики в сфере образования различают три основных направления децентрализации: (1) деконцентрацию, (2) использование рыночных механизмов и (3) внедрение различных форм пар-

\footnotetext{
${ }^{4}$ Индекс РФ существенно ниже, чем в группе наиболее «автономизированных» стран, но приблизительно аналогичен, а в некоторых случаях выше, чем у федерации ЕC. См.: OECD, PISA 2009 Results. - P. 70.
}

ситипативной демократии, в рамках которой жители муниципалитета, региона (провинции, земли, округа и т.п.), страны сами разрабатывают и применяют механизмы, позволяющие им управлять системой образования на своей территории ${ }^{5}$.

Деконцентрацчия. В большинстве стран, использовавших механизм деконцентрации, предоставляющих большую автономию школьным учреждениям и управляющим ими органам на местном уровне, это привело к расширению автономии школ в вопросах распределения и использования ресурсов, а также разработки учебных планов и программ, т.е. формирования профиля школ. В качестве вариантов деконцентрации в странах Европейского Союза можно привести мероприятия в Чешской республике, Нидерландах, Великобритании, где автономия школ расширялась в обоих направлениях. В Венгрии, Швеции, Болгарии она коснулась в основном ресурсного обеспечения.

Во многих случаях расширение автономии школ сопровождается более высокими требованиями к отчетности и повышением ответственности школ за качество предоставляемых ими услуг. Целью в данном случае является не только более выраженная легитимация решений, принимаемых на уровне школы, но и повышение качества образования в целом. Как подчеркивается в изданном в 2011 году отчете OECD[6], выявить прямую взаимосвязь между автономией школ и качеством образовательной системы в целом в конкретных странах пока достаточно сложно: «На уровне стран в целом результаты деятельности образовательной системы тем лучше, чем

\footnotetext{
${ }^{5}$ См.: Waslander $S$. et al. Markets in Education. An analytical review on empirical research on market mechanisms in education. - OECD Education Working Papers. - 2010. - No. 52, OECD Publishing, DOI: 10.1787/5km4pskmkr27-en.; Ball, S.J. Privatising education, privatising education policy, privatising educational research: network governance and the ,competition state //. Journal of Education Policy. - 2009. - 24(1). - pp. 83-99; Hudson, C. Governing the governance of education: The state strikes back? // European Educational Research Journal. - 20076(7). - pp. 266-282; San Antonio, D.M., Gamage, D.T. PSALM for empowering educational stakeholders: Participatory school administration, leadership and management //. International Journal of Educational Management. - 2007. - 21(3). - pp. 254265; Fung, A., Wright, E. O. Deepening democracy: Innovations in empowered participatory governance //. Politics and Society. - 2001. - 2. - pp. 95-41.
} 


\section{Политика и общество $2(98) \cdot 2013$}

большее количество школ имеет право определять и разрабатывать свои учебные планы и программы, а также механизмы оценки результатов обучения. Эта взаимосвязь сохраняется также после корректировки результатов в соответствии с размером валового национального продукта. Эта взаимосвязь наблюдается даже в тех случаях, когда результаты отдельной школы не коррелируют с уровнем ее автономии в сфере разработки учебного плана. В противоположность этому, на общенациональном уровне не выявляется взаимосвязь между автономией в сфере распоряжения ресурсами и качеством образования».

Использование рыночных механизмов: расширение возможностей выбора для родителей. Данный вариант децентрализации призван расширять спектр учреждений, среди которых могут делать выбор родители, а также обеспечивать конкуренцию между школами. При этом чаще всего используется отмена фиксированных территорий, закрепленных за школами, внедрение ваучерных программ и открытие чартерных школ ${ }^{6}$. Считается, что родители, обладая правом выбора, способны лучше всего выбрать школу или форму получения образования для своего ребенка.

В последние годы появился ряд исследований, которые приходят к противоречивым выводам о возможности широкого использования рыночных механизмов, т.к. демонстрируемые незначительные улучшения (обычно в баллах по чтению, реже - по математике) соседствуют с данными об усилении сегрегации в школьной системе, как с социальной токи зрения (более и менее обеспеченные семьи), так и о большем расхождении

\footnotetext{
${ }^{6}$ См.: Waslander $S$. et al. Markets in Education. An analytical review on empirical research on market mechanisms in education.; Faubert, V. School evaluation: Current practices in OECD countries and a literature review. - OECD Education Working Papers. - 2009. - o. 42. Пояснения требует термин «чартерная школа», употребляемый в русскоязычных текстах. Термин происходит от английского слова «charter» - хартия, грамота. Содержание понятия «чартерная школа» состоит в наличии у школы собственной «хартии, устава», обеспечивающего учреждению максимальную автономию по всем аспектам управления учебным и воспитательным процессом. Такой тип школы широко распространен в США. При этом, чартерная школа остается публичной, за ней сохраняется государственное или муниципальное финансирование, она отчитывается за результаты образовательной деятельности.
}

возможностей для более способных и менее способных учащихся ${ }^{7}$.

Парситипативная демократия: усиление влияния членов сообществ (муниципальных, специальных, региональных, национальных). Данный вариант требует расширения возможностей прямого участия граждан в процессе определения и реализации политики в сфере образования на местном уровне. Главными механизмами являются при этом своеобразная «фора», которая предоставляется родителям, членам общественных организаций, объединений работодателей для того, чтобы они могли сформировать предварительно свою позицию с учетом местных потребностей и непосредственно участвовать в принятии решения; предоставление грантов структурами местного уровня (дополнительных ресурсов для повышения возможностей получения образования индивидуумами или группами); а также введение должности специалиста, стимулирующего активность родителей и различных групп в выявлении потребностей в обучении и организации развития в требуемом направлении[7]. Концепция парситипативной демократии в образовании включает также создание различных комиссий и комитетов, в которые активно вовлекаются ученики, родители, учителя и другие заинтересованные жители.

Исследования влияния демократических форм на эффективность и результативность образования демонстрирует положительные тенденции, однако, не столь выраженные, как в случае использования деконцентрации или рыночных механизмов. В общем виде считается, что в неформальных связях более ярко выражается политическая воля граждан, лучше учитываются потребности, т.к. включающиеся в процесс непрофессионалы действуют менее формализовано, чем специалисты, работающие в сфере образования или чиновник органов управления образованием ${ }^{8}$.

\footnotetext{
${ }^{7}$ Cм.: Waslander $S$. et al. Markets in Education. An analytical review on empirical research on market mechanisms in education; 9; Ball, S.J. Privatising education, privatising education policy, privatising educational research: network governance and the „competition state”.

${ }^{8}$ Banerjee, $A$. et al. Can information campaigns raise awareness and local participation in primary education? // Economic and Political Weekly. - 2007. - 14 April. - pp. 1365-1372; San Antonio, D.M., Gamage, D.T. PSALM for empowering educational stakeholders: Participatory school administration, leadership
} 
Как показывает проведенный выше анализ различных направлений децентрализации, она не огранивается только передачей полномочий на более низкие уровни управления, но предусматривает также изменение механизмов взаимодействия школы с различными акторами, участвующими в процессе. В целом можно констатировать, что правительства, избравшие данный путь, значительно расширили автономию школ, повысили восприимчивость к изменению потребностей в сфере образования и конкуренцию между школами, а также предоставили возможность влиять на сферу образования родителям и другим группам, активно заинтересованным в принятии решений в рамках политики в сфере образования. Параллельно им пришлось искать и внедрять новые механизмы отчетности с целью усиления ответственности школ и других управляющих структур нижнего уровня за качество образования в рамках принимаемых ими решений.

Отчетность школ в новых условиях становится не только средством соблюдения закона, но и инструментом повышения качества образования, т.к. будучи обязанными соблюдать высокие стандарты, руководители школ и учителя стремятся к повышению качества своей работы. В целом специалисты выделяют 3 основные цели, преследуемые различными формами отчетности школ:

1. Легитимация принимаемых ими решений в ходе выявления их соответствия действующим законам.

2. Ответственность за качество предоставляемых ими услуг в сфере непосредственно качества образования (эффективность деятельности), цены использованных финансовых ресурсов (экономичность), равенства и доступа образования для всех.

3. Положительная динамика развития в сфере качества образования и более качественного использования ресурсов[8].

В связи с расширением автономии школ и повышении интереса к качеству образования в сфе-

and management; Cheng, Y.C., Cheung, W.M. Profiles of multilevel self-management in schools // The International Journal of Educational Management. - 2003. - 11(3). - pp. 100-115; Marzano, R.J. What Works in Schools? Translating Research into Action. Association for Supervision and Curriculum Development - ASCD, 2003. ре отчетности произошли значительные сдвиги в двух направлениях: с одной стороны, к традиционной вертикальной ответственности (перед вышестоящими уровнями управления) присоединилась горизонтальная ответственность, которая в наиболее общем виде может быть охарактеризована как переход от отчетности за расходование ресурсов к детальному контролю результатов деятельности (school performance accountability) [9]. При достаточно широком распространении в странах ЕС и ОЭР, эта форма контроля значительно варьируется в различных системах: наиболее часто используется тестирование, широко представлено издание национальных отчетов о развитии образования, а также в ряде стран используется вручение национальных премий и публичное порицание[9].

Вторым существенным сдвигом в сфере отчетности и контроля стал переход от контроля одного конкретного аспекта деятельности в сфере образования и включения одного контролирующего субъекта к многоаспектному контролю, осуществляемому множественными субъектами. Обеспечение эффективности и результативности такого контроля оказалось достаточно сложной теоретической и практической проблемой. Осуществление только контроля за результатами деятельности школ не позволяет учитывать такие важнейшие аспекты как процесс социализации, интеграцию общества, развитие профессиональных ресурсов. В ходе многоаспектного контроля в процесс включаются ученики, учителя, руководство школы, родители, представители муниципалитетов, работодатели и другие заинтересованные лица. Сам процесс при этом включает три основных направления контроля: (1) легитимацию стратегии школы и принимаемых ею решений (правильно ли направлено развитие; (2) легитимацию качества образования (насколько качественно реализуется стратегия); (3) улучшение качества предоставляемых услуг. Расширение процесса контроля в данном направлении позволяет сформировать холистический взгляд на систему образования и отслеживать реальную динамику ее развития9

9 Cм.: Morris, A. Student standardised testing: current practices in OECD countries and a literature review. - OECD Education Working Papers. - 2011. - No. 65; Rosenkvist, M.A. Using student test results for account- 


\section{Политика и общество 2 (98) • 2013}

Таким образом, необходимо дополнять контроль за результатами деятельности школ многоаспектным контролем для обеспечения прозрачности всех процессов в системе путем открытия ее внутренних механизмов и их функционирования для анализа их широкой общественностью. Однако при введении таких механизмов правительствам необходимо учитывать риск перегрузки информацией, что ведет к затруднению принятия решений или принятия их на нерациональной основе. Объем и структуру информации при введении многоаспектной оценки правительствам необходимо согласовывать с школьными учреждениями и другими специалистами, участвующими в процессе.

Подводя итог анализа взаимосвязи между уровнем автономии школьного учреждения (по двум основным направлениям - (1) самостоятельность в определении содержания образования и контроля за результатами учащихся, (2) полномочия по распоряжению ресурсами), системой отчетности школ за результаты деятельности и качеством школьного образования в целом, европейские исследователи делают вывод: «Автономия школ в области распределения ресурсов, как правило, улучшает результаты обучения в системах образования, где публикуются результаты работы школьных учреждений, в противном случае расширение автономии дает скорее отрицательный эффект. Это позволяет констатировать, что расширении автономии не может быть изолированным направлением политики в сфере образования, а входит в комплекс мероприятий по совершенствованию процесса управления в сфере образования» ${ }^{10}$.

ability and improvement: A literature review. - OECD Education Working Papers. - 2010. - No. 54; Feng, L., et al School accountability and teacher mobility. -. Working Paper of Calder Urban Institute. - 2010. - 47; Resnick, L.B. Making accountability really count//Educational Measurement: Issues and Practice.-Spring2006.-25(1).-pp.33-37; Kane, J.K., Staiger, D.O. The promise and pitfalls of using imprecise school accountability measures // Journal of Economic Perspectives. - 2002. - 16(4). - pp. 91-114; Ladd, H. F., Zelli, A. School-based accountability in North Carolina: The responses of school principals // Educational Administration Quarterly. - 2002. - 38(4). - pp. 494-529.

${ }^{10}$ В цифрах это корреляция выглядит следующим образом: в странах, где $100 \%$ школ публикуют результаты своей работы, ученики школ с более высоким уровнем ав-

\section{Библиография:}

1. ОЭСР. Равенство и качество образовательных услуг: поддержка слабоуспевающих учащихся и проблемных школ. - ОЭСР. Лучшая политика для лучшей жизни. Образование. 09.02. 2012 // http://oecdru.org/obraz.html

2. Bruns, B., Filmer, D., Patrinos H.A. Making on accountability reforms. Washington, D.C.: The World Bank, 2011.

3. OECD. School autonomy and accountability: Are they related to student performance? // PISA in Focus - 9, 2011. - P.2 // http://www.oecd.org/pisa/pisainfocus-monthlypolicy-orientednotes.htm

4. Hanushek E. A., Link S., Woessmann L. Does School Autonomy Make Sense Everywhere? Panel Estimates from ISA / Discussion Paper Serious IZA DP N 6185, $2011 / /$ http://www.nber. org/papers/w 17591

5. Waslander, S., Pater, C/. van der Waide, M. Markets in education: An analytical review of empirical research on market mechanisms in education. - OECD Education Working Papers, No. 52, OECD Publishing, DOI: 10.1787/5km4pskmkr27-en.2010.

6. OECD. School autonomy and accountability: Are they related to student performance? // PISA In Focus. - 2011. - No. 9. - p. 2.

7. Ranson, S. The changing governance of education //. Educational Management Administration and Leadership. - 2008. - 36(2). - pp. 201-219.

8. Geijsel, F. P. et al Data feedback for school improvement: The role of re-searchers and school leaders // The Australian Educational Researcher. -2010 . - 37(2). - pp. 59-75.

9. OECD. Education at a Glance 2011: OECD Indicators. - OECD Publishing, 2011.

тономии на 5 пунктов опережают учеников школ с меньшим уровнем автономии в тестах по чтению. В странах, где школы не публикуют результаты, ученики более автономных в сфере распределения ресурсов школ отстают от учеников менее автономных учреждений на такое же количество пунктов. Этот урок может быть достаточно поучительным для России, где автономия часто понимается как полная самостоятельность и независимость от целостной системы образования. Результаты исследований ОЭСР см.: OECD. School autonomy and accountability: Are they related to student performance? // PISA in Focus - 9, 2011. - P.2 // http://www.oecd.org/pisa/pisainfocus-monthly policy-orientednotes.htm 
Гуманитарные проекты

\section{References (transliteration):}

1. OESR. Ravenstvo i kachestvo obrazovatel'nykh uslug: podderzhka slabouspevayushchikh uchashchikhsya i problemnykh shkol. - OESR. Luchshaya politika dlya luchshey zhizni. Obrazovanie. 09.02. 2012 // http://oecdru.org/obraz.html

2. Bruns, B., Filmer, D., Patrinos H.A. Making on accountability reforms. Washington, D.C.: The World Bank, 2011.

3. OECD. School autonomy and accountability: Are they related to student performance? // PISA in Focus - 9, 2011. - P.2// http://www.oecd.org/pisa/pisainfocus-monthlypolicy-orientednotes.htm

4. Hanushek E. A., Link S., Woessmann L. Does School Autonomy Make Sense Everywhere? Panel Estimates from ISA / Discussion Paper Serious IZA DP N 6185, $2011 / /$ http://www.nber. org/papers/w17591
5. Waslander, S., Pater, C/. van der Waide, M. Markets in education: An analytical review of empirical research on market mechanisms in education. - OECD Education Working Papers, No. 52, OECD Publishing, DOI: 10.1787/5km4pskmkr27-en.2010.

6. OECD. School autonomy and accountability: Are they related to student performance? // PISA In Focus. - 2011. - No. 9. - r. 2.

7. Ranson, S. The changing governance of education //. Educational Management Administration and Leadership. - 2008. - 36(2). pp. 201-219.

8. Geijsel, F. P. et al Data feedback for school improvement: The role of re-searchers and school leaders // The Australian Educational Researcher. $-2010 .-37(2)$. - pp. 59-75.

9. OECD. Education at a Glance 2011: OECD Indicators. - OECD Publishing, 2011. 